\title{
A Classification Approach for Power Distribution Systems Fault Cause Identification
}

\author{
Le Xu, Student Member, IEEE, and Mo-Yuen Chow, Senior Member, IEEE
}

\begin{abstract}
Power distribution systems play an important role in modern society. When distribution system outages occur, fast and proper restorations are crucial to improve the quality of services and customer satisfaction. Proper usages of outage root cause identification tools are often essential for effective outage restorations. This paper reports on the investigation and results of two popular classification methods: logistic regression (LR) and artificial neural network (ANN) applied on power distribution fault cause identification. $\mathrm{LR}$ is seldom used in power distribution fault diagnosis, while ANN has been extensively used in power system reliability researches. This paper discusses the practical application problems, including data insufficiency, imbalanced data constitution, and threshold setting that are often faced in power distribution fault cause identification problems. Two major distribution fault types, tree and animal contact, are used to illustrate the characteristics and effectiveness of the investigated techniques.
\end{abstract}

Index Terms-Artificial neural network (ANN), classification, data insufficiency, fault cause identification, imbalanced data, logistic regression (LR), power distribution systems, threshold setting.

\section{INTRODUCTION}

A S THE RETAIL part of utilities, power distribution systems aim at providing reliable, economical, and safe supply of electricity to the users. However, power distribution systems are geographically dispersed, and under various dynamic operating environments, they can be significantly affected by various faults in terms of power reliability and quality of services, such as equipment failure, animal contacts, trees, lightning, etc.

In order to improve system safety and reliability, power distribution management systems should have fast and proper response to power outages. Currently, when a power outage is reported to the control center by customers, a typical response is that the operators estimate the probable outage location based on available information and call up their operation and maintenance (O\&M) crews to fix the problem. For safety reasons, many utilities do not restore the distribution system until they have found the outage cause. Sometimes, the outage cause cannot be found in the fault location. For example, a squirrel jumped into a distribution transformer and shorted the phase to ground wires. However, a bird took away the dead squirrel body; thus, the fault cause evidence could not be found anymore. The lineman needs to walk down the line, which can

Manuscript received March 16, 2005; revised September 3, 2005. This work was supported by the National Science Foundation under Grant ECS-0245383. Paper no. TPWRS-00161-2005.

The authors are with Department of Electrical and Computer Engineering, North Carolina State University, Raleigh, NC 27695 USA (e-mail: xule@ieee.org; chow@ncsu.edu).

Digital Object Identifier 10.1109/TPWRS.2005.861981 be miles, in an attempt to find the outage evidence (e.g., burn marks on the pole for possible lightning faults, dead animal bodies for possible animal faults) and to ensure everything is safe (e.g., no down distribution lines) before they re-energize the system. For some of the outage causes (e.g., tree fault), they may need to call in the control center to dispatch appropriate crews (e.g., tree crew) to remove the fallen trees in order to restore the system. The whole process may take tens of minutes to hours. Utilities have been making substantial effort to expedite the restoration procedure; for example, sectionalizing the feeders to quickly restore as many customers as possible on power by putting every subsection back once workers assure its proper working instead of restoring the whole section at the same time [1]. Many different methods, such as Bayesian network [2], support vector machine [3], and current pattern matching [4], have been investigated to locate the fault.

This paper focuses on the outage root cause identification; reasonable fault cause estimation can provide workers with useful information to narrow down the searching areas so as to help to expedite the restoration procedure. With good fault cause estimation, the control center can inform the lineman to focus on what evidence to look for. For example, if the fault is likely to be caused by animals, then the control center will ask the lineman to focus on dead animal bodies on the top of distribution transformer or the ground close to the distribution poles, with less emphasis on burn marks on the distribution equipment that can be caused by lightning. The control center can also dispatch appropriate crew earlier to restore the system. For example, if the fault cause is estimated to be a tree cause fault, then the control center will prepare and be ready to dispatch the tree crew to restore the system. Thus, a good estimate on the fault cause can significantly reduce the "trouble shooting" time, thus reducing the restoration time and consequently improving the system reliability.

Power distribution fault cause identification can be viewed as a classification problem in that operators try to categorize the reported outage into one of the existing fault cause classes carefully arranged by domain experts, while a typical classification problem aims to extract models from training data with known class labels to predict the categories of the test data of which the class labels are unknown. Many methods use current and voltage for fault classification [5], [6]. With the development of data mining techniques, some research has investigated fault diagnosis using the extracted patterns from historical outage data: An approach using rough set theory to mine the historical fault data has been proposed in [7].

In this paper, a power distribution fault cause classifier (PDFCC) has been developed to address the fault cause identification problem in the power distribution systems. The 
investigation and results of two classification methods, logistic regression (LR) and artificial neural network (ANN) applied to mine the historical outage data for power distribution fault cause classification, are presented. A regular LR is a parametric model used to analyze the problems with dichotomous dependent variables; it has been widely applied in medicine and biology areas but not in power system fault diagnosis. On the other hand, ANN is a nonparametric method that has been extensively used in various research and application areas, including power systems [8], [9]. Evaluation criteria of the goodness of the PDFCC include: correct classification rate (CCR); true positive rate; true negative rate; and geometric mean. This paper discusses the practical application problems like data insufficiency, imbalanced data constitution, and threshold setting that are often faced in power distribution fault cause identification problems. Tree and animal contact, which are two of the top customer interruption causes for most utilities, are used in this paper to illustrate the characteristics and effectiveness of the investigated techniques.

\section{Power Distribution Fault Data Preprocessing}

This paper uses Duke Energy power distribution outage data to illustrate the proposed outage root cause identification scheme. Duke Energy has a detailed power distribution fault data collection system. Every time a fault current is detected in the distribution system as a result of the activation of protective devices (e.g., a circuit breaker, a fuse), the information related to that distribution fault is recorded into this database as one record entry. Each fault record has 33 information fields; based on Duke Energy senior distribution engineers' suggestions, six of them are considered as containing most essential and influential information for tree-caused faults and animal-caused faults [10], [11] and are selected to be used in this paper. These six factors have been tested to be statistically significant influential factors [10]. These factors are circuit ID number (CI), weather condition (WE), season (SE), time of day (TD), number of phases affected (NP), and protective devices activated (PD). Each factor and its contents are represented in set notations; one example is

$\mathrm{WE}=\{$ fair, cold, rain, wind, wind \& lightning,

lightning, hail, snow, ice, hot, storm\}.

Like the factor of weather condition, most of these six input factors are nonnumerical variables; they are transformed into numerical nominal variables so that they can be easily represented in the training set. For instance, fair weather is represented by number 0 ; cold is denoted as 1 ; and so on. The numerical data here are used as nominal values rather than continuous values to simply serve as a "tag" to represent the weather condition.

In addition, the attribute cause that records the root cause of the outage is also used. It is entered by the crew after finding the actual outage cause during the restoration process. This paper uses tree-caused faults and animal-caused faults as prototypes; so we categorize the root causes into three classes: $\{$ tree-caused fault (T), animal-caused fault (A), other causes $(\mathrm{O})\}$. The
PDFCC proposed in this paper will classify an outage into one of these three classes.

The Duke Energy outage data consist of outage records from its 32 service regions in North Carolina and South Carolina ranging from 1994 to 2002 . Based on domain experts' suggestions, we take different geographical features and system status into account and select seven regions as reasonable representations of Duke Energy's service areas: Chapel Hill (CHPL), Clemson (CLEM), Durham (DURH), Greenville (GREE), Hickory (HICK), Lancaster (LANC), and Winston-Salem (WINS). These seven regions cover metropolitan areas, cities, towns, rural areas, and wooded areas and also embody both old systems and new systems.

\section{ClassificAtion Methods}

There are many well-established classification methods; empirical studies show that many algorithms have sufficiently similar accuracies such that the differences between those algorithms are statistically insignificant [12]. It is important for PDFCC, which aims at solving practical power distribution fault cause identification, to select a proper classification method considering the practical application issues like ease of implementation and computational requirements in addition to accuracy. LR and ANN are two classification methods widely used in numerous applications; they can be easily and quickly implemented using several existing software or toolboxes such as SAS and MATLAB. In this paper, we employ these two methods to mine the historical outage data and extract distribution fault cause classification models from the data.

\section{A. $L R$}

LR is a well-known statistic method to analyze problems with a dichotomous (binary) dependent variable. The dichotomous classification problems were commonly analyzed by ordinary least-squares linear regression, which, in fact, often did a reasonably good job. However, a dichotomous dependent variable violates the normality assumption and homoscedasticity assumption of the linear regression model [13]. Consequently, researchers seek to develop alternative methods by trying to predict the probability of a case being classified into one category of the dependent variable as opposed to the other; for example, if we only consider the animal-caused fault, we want to estimate the probability of an outage being caused by animal contact as opposed to the probability of not being caused by animal. Assuming that we use 1 and 0 to represent the two possible values of the dependent variable, the problem can be formulated as

$$
P(Y=1)=a+b_{1} x_{1}+b_{2} x_{2}+\cdots+b_{6} x_{6}
$$

where $Y$ is the dependent variable (e.g., animal-caused fault) $x_{k}, k=1,2, \cdots, 6$ is an independent variable (e.g., CI, WE, NP, $\mathrm{PD}, \mathrm{SE}, \mathrm{TD}), a$ and $b_{1}, b_{2}, \cdots, b_{6}$ are parameters to be identified based on the training data.

Probability value must be between 0 and 1 , but the predicted value from the independent variables $x_{1}, x_{2}, \cdots, x_{6}$ in the righthand side of (1) may be less than 0 or greater than 1 . In order 
to resolve this problem, the probability is transformed to odds, which refers to the ratio of the probability that the event will occur to the probability that the event will not occur

$$
\operatorname{odds}(Y=1)=\frac{P(Y=1)}{P(Y=0)}
$$

The odds does not have a maximum value; however, it has a minimum value bounded by zero. The odds is always nonnegative because it is the ratio of two probability values. A further transformation using the natural logarithm of the odds is performed. The natural logarithm of the odds is called logit, which can be any number between plus and minus infinity

$$
\begin{aligned}
\operatorname{logit}(Y=1) & =\ln [\operatorname{odds}(Y=1)] \\
& =\alpha+\beta_{1} x_{1}+\beta_{2} x_{2}+\cdots+\beta_{6} x_{6}
\end{aligned}
$$

where $\alpha$ and $\beta_{1}, \beta_{2}, \cdots, \beta_{6}$ are parameters to be identified by the training data.

The model specified in (3) is the LR model; its unknown coefficients $\alpha$ and $\beta_{1}, \beta_{2}, \cdots, \beta_{6}$ are estimated with maximum likelihood [13] parameter estimation method using the training data.

Equation (3) can be easily solved for the probability of the observed case being in the category of interest as shown in

$$
P(Y=1)=\frac{1}{\left(1+e^{-\left(\alpha+\beta_{1} x_{1}+\beta_{2} x_{2}+\cdots+\beta_{6} x_{6}\right)}\right)} .
$$

When LR is applied as a classification method to separate patterns between two classes, the unknown parameters $\alpha$ and $\beta_{1}, \beta_{2}, \cdots, \beta_{6}$ of the LR model are estimated from the training data first; then the probability $P(Y=1)$ of a test case is calculated using (4); in the end, the class label is assigned to that test case by comparing the calculated probability with a predefined threshold.

\section{B. ANN}

A neural network is a set of interconnected simple processing elements called nodes or neurons, where each connection has an associated weight. A neural network can exhibit certain behavior or achieve desired input-output mapping with specified set of weights stored in the connections between nodes; therefore, we can train the neural network to do a particular job by adjusting the weights on each connection [14].

The processing element neurons are usually organized into series of layers. A neural network typically consists of three or more layers. The data are presented to the input layer by which the data are passed into the network. The data from the input layer arrive at the intermediate layer or the hidden layer with the associated connection weights. The hidden layer takes in the weighted inputs and calculates the outputs by the hidden neurons' transfer functions. The calculated outputs are fed to the next hidden layer in turn (if there are more than one) or the output layer. The output layer then generates the results representing the mapping from the given input data. There is no clear rule to determine the number of neurons in each layer, which is generally done by educated trial and error. The network of which none of the weights are fed backward is called a feed-forward neural network.
Once the network structure has been determined, the weight of each connection in the network is to be learned from the training data. The most popular learning algorithms is backpropagation. The network connection weights are initialized to small random numbers, and then the inputs are propagated forward by activating the neuron transfer functions and calculating the outputs of each layer in turn to calculate the network output; afterwards, the error between the actual network output and desired response is propagated backward to update the network parameters in order to minimize the error of the network prediction. Back-propagation iteratively processes the training samples through the input forward propagation and error backward propagation until a specified accuracy or specific terminating conditions are satisfied.

ANN technique has been applied to many different areas, such as pattern recognition and classification. When a neural network is used as a classification technique, its operation involves two steps: learning and recall. In the learning phase, all the weights in the network are adjusted to adapt to the patterns of the training data in accordance with the learning algorithm; in the recall phase, the network produces responses of the inputted test data based on learned network parameters.

\section{PDFCC}

The flowchart of the PDFCC developed in this paper for power distribution fault cause identification is shown in Fig. 1.

Since we consider two fault causes in this paper, the PDFCC consists of two parallel branches. These two branches are identical in terms of structure and operating mechanism, but they work on different fault causes. Branch A classifies the input fault as either an animal or nonanimal fault, while branch T simultaneously works on tree faults. If we consider $\mathrm{N}$ fault causes, then we will have $\mathrm{N}$ parallel branches. The outputs from two individual branches are mediated by the decision fusion module when conflict happens; it will be discussed in a later section.

\section{A. System Inputs}

The input data to PDFCC consist of the six selected information factors introduced in Section II

$$
\begin{array}{r}
\mathbf{X}=\left\{\left(x_{1}, x_{2}, x_{3}, x_{4}, x_{5}, x_{6}\right) \mid x_{1} \in C I, x_{2} \in W E,\right. \\
\left.x_{3} \in S E, x_{4} \in T D, x_{5} \in N P, x_{6} \in P D\right\} .
\end{array}
$$

\section{B. Data Preprocessor (Animal/Tree)}

Four statistical measures have been proposed in [11] to examine the outage data from different perspectives. Likelihood measure, one of the developed measures, indicates the probability that an outage is caused by a specific fault under a specified condition. This measure can be expressed as

$$
L_{i, j}=\frac{N_{i, j}}{N_{j}}
$$

where $L_{i, j}$ is the likelihood measure of fault $i$ given event $j$, $N_{i, j}$ is the number of outages caused by fault $i$ under event $j$, and $N_{j}$ is the total number of outages under event $j$. 


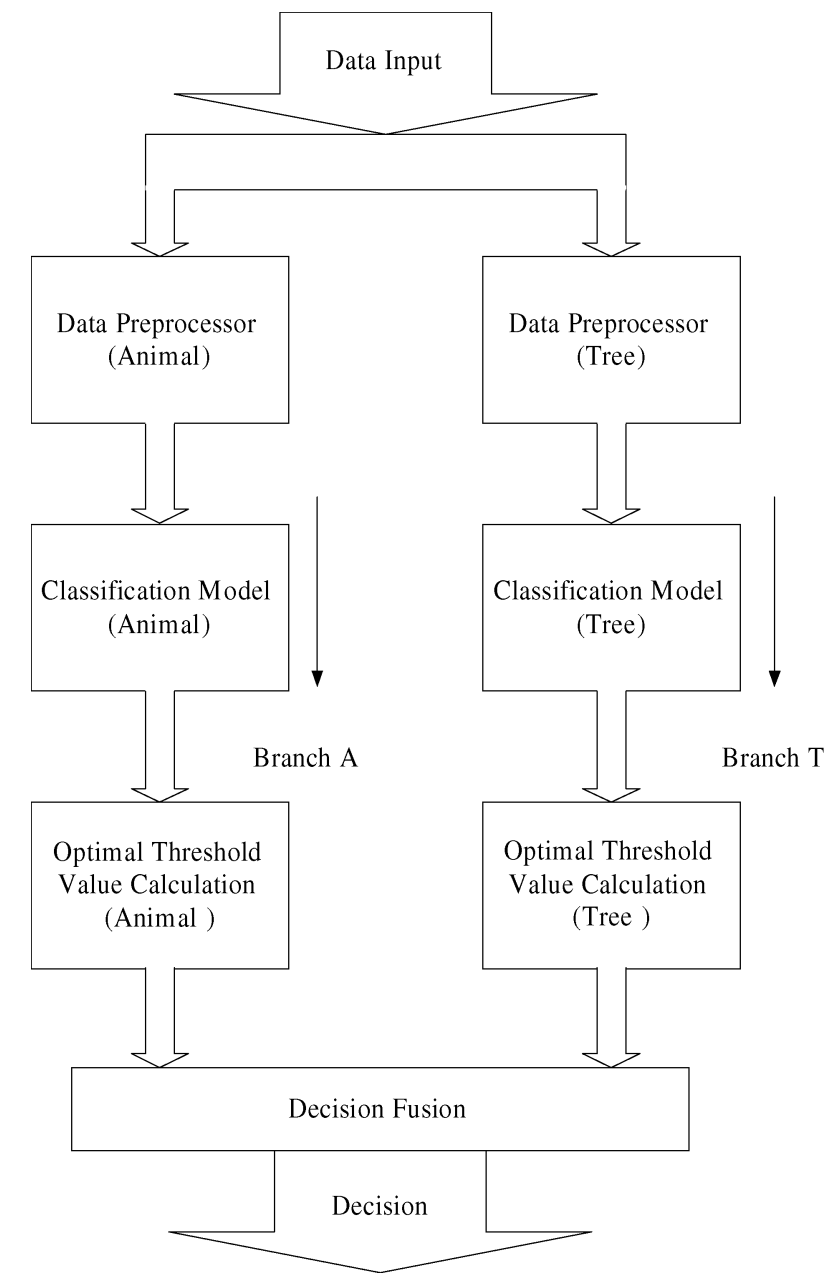

Fig. 1. Flow chart of PDFCC.

For example, if $i$ indicates a tree-caused fault and $j$ indicates icy weather conditions, then the likelihood measure $L_{i, j}$ shows the conditional probability of the observed outage being caused by a tree given icy weather conditions. Likelihood measure can provide useful information for fault cause identification, so it is logically used as the inputs to the classification methods for outage cause identification. Equation (5) indicates that likelihood measure is related to both fault type and event; the likelihood measure for tree-caused faults and that for animal-caused faults are different, even given the same event. Thus, we need to utilize two branches to deal with two fault types, respectively.

The data preprocessor modules in both branches take in the input $\mathbf{X}$, calculate the corresponding likelihood measures $L_{i, j}$ with respect to different fault causes, and then pass $L_{i, j}$ to the classification modules.

\section{Classification Module (Animal/Tree)}

We use LR and ANN in this module in order to compare these two popular classification techniques for power distribution fault cause identification under the same environments. Both methods implement a mapping from the likelihood measures $L_{i, j}$ as defined in (5) to a probability value $P_{i}$

$$
f: L_{i, j} \rightarrow P_{i}
$$

1) $L R$ : For the branch of fault $i, i \in\{$ animal,tree $\}$, the likelihood measures $L_{i, j}$ from the corresponding classification module are the independent variables of the LR model, and the probability $P_{i}$ is the dependent variable.

2) ANN: The inputs and output are in correspondence with the independent variables and dependent variable of LR model. A three-layer feed-forward network with one hidden layer of 40 nodes is used in this paper (which has been chosen through extensive simulation of different numbers of hidden nodes.) Fivefold cross-validation is used to prevent overfitting.

\section{Optimal Threshold Value Calculation (Animal/Tree)}

The output of the classification module $P_{i}$ is the probability of the observed input data $\mathbf{X}$ being caused by fault $i$; in order to categorize the observed outage into one class or the other, a threshold is needed. With the selected threshold $\zeta_{i}$ and calculated probability $P_{i}$ from the classification module, a class label can be generated: animal-caused fault or nonanimal-caused for branch A, tree-caused fault or nontree-caused for branch T, and mathematically represented as

$$
C_{i}= \begin{cases}1, & P_{i} \geq \zeta_{i} \\ 0, & P_{i}<\zeta_{i}\end{cases}
$$

where $C_{i}$ is the class label and " 1 " stands for the outage caused by fault $i$, while " 0 " stands for the outage not caused by fault $i$.

Usually the threshold $\zeta_{i}$ is set as 0.5 (for $0 \leq P_{i} \leq 1$ ) for an ideal case (e.g., plenty of data and they are all independent and identically distributed). However, the outage data used are imbalanced. The average proportion of tree-caused faults is only $23.6 \%$ and animal-caused faults is $13.2 \%$, while other faults account as much as $63.2 \%$; therefore, we adjusted $\zeta_{i}$ to an optimal value rather than use the fixed value of 0.5 to compensate the asymmetry of the sample composition in the data in order to maximize the correct classification rate, as shown in Fig. 2.

Fig. 2 shows an example of the distributions of the calculated $P_{\text {tree }}$ for both tree-caused and nontree-caused faults, represented by $f_{t}$ and $f_{n-t}$, respectively. Note that these two curves $f_{t}$ and $f_{n-t}$ are asymmetric. The area under curve $f_{n-t}$, denoted as $S_{n-t}$, shows the number of nontree-caused faults and the area under curve $f_{t}$, denoted as $S_{t}$, indicates the number of tree-caused faults. Lines $\zeta=\mathrm{a}=0.50$ and $\zeta=\mathrm{b}=0.55$ are the thresholds (decision lines). All the cases with $P_{\text {tree }} \geq \zeta$, $\zeta \in\{a, b\}$ are categorized as tree faults, and all the cases with $P_{\text {tree }}<\zeta$ are classified as nontree faults. We want to maximize $S_{n-t, P_{i}<\zeta}$ and $S_{t, P_{i} \geq \zeta}$ and minimize $S_{t, P_{i}<\zeta}$ and $S_{n-t, P_{i} \geq \zeta}$ by selecting an optimal threshold; obviously, threshold $b$, which passes the intersection of $f_{t}$ and $f_{n-t}$, is the optimal one.

When the threshold value increases from 0 to $1, S_{t, P_{i}<\zeta}$ keeps increasing, while $S_{n-t, P_{i} \geq \zeta}$ always decreases; it indicates that the misclassification of tree-caused faults is always increasing and the misclassification rate of nontree-caused fault is decreasing. It is obvious to see from Fig. 2 that the decrease of $S_{n-t, P_{i} \geq \zeta}$ is much faster than the increase of $S_{t, P_{i}<\zeta}$ before the threshold reaches the intersection of two lines and the other way around after the intersection; therefore, the overall correct classification rate goes up to a maximum point first and then goes down, as shown in Fig. 3. 




Fig. 2. Threshold selection for imbalanced data.

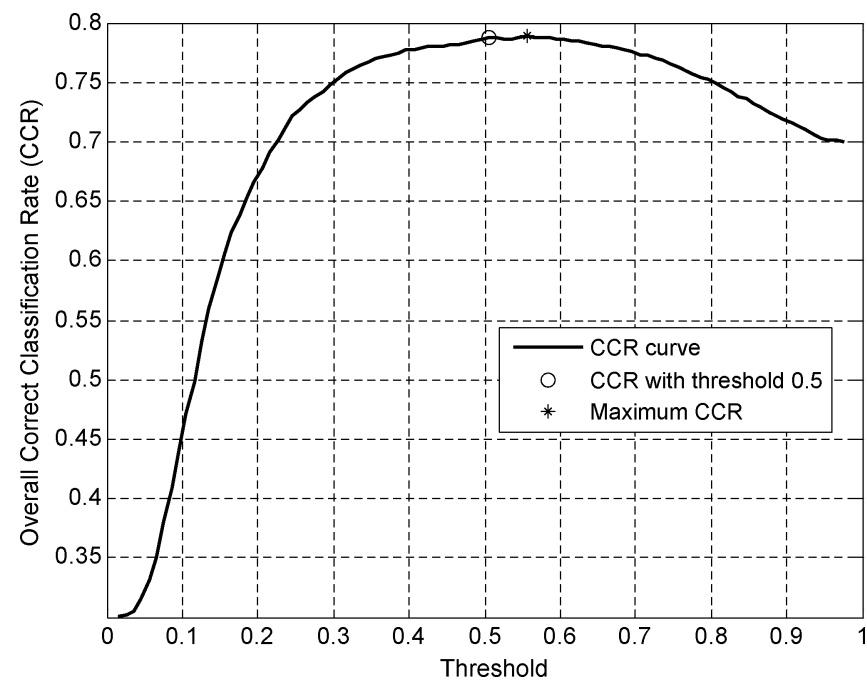

Fig. 3. Correct classification rate curve with different threshold settings.

Fig. 3 shows the CCR of tree-caused fault data in the Lancaster region. The peak value of CCR is 0.7887 achieved by a threshold value of about 0.55 . When the threshold is 0.5 , the CCR is 0.7869 . When the threshold is 0 , all outages are classified as tree-caused faults, which account for about $30 \%$ of the total outages, so the CCR is only about 0.3 . When the threshold is 1 , every single outage is classified as a nontree-caused fault, accounting for about $70 \%$ of the total outages, so the CCR is about 0.7 .

Fig. 4 shows the sensitivity of CCR (Sensitivity = $\left.\frac{\partial \mathrm{CCR}}{\text { threshold }} \cong \frac{\Delta \mathrm{CCR}}{\Delta \text { threshold }}\right)$ at different threshold values. When the threshold is selected around 0.55 (the optimal point), the sensitivity is close to 0 , which indicates the classification performance is relatively not sensitive to the perturbation in the selected threshold value. When the threshold is selected far away from the optimal point, say, 0.1, a small change in the threshold value will cause a large change in the overall classification accuracy.

\section{E. Decision Fusion}

Each branch only considers the patterns associated with either a tree- or an animal-caused fault and provides the estimation whether the inputted outage is caused by its designated fault cause: tree or animal. When the estimations from two

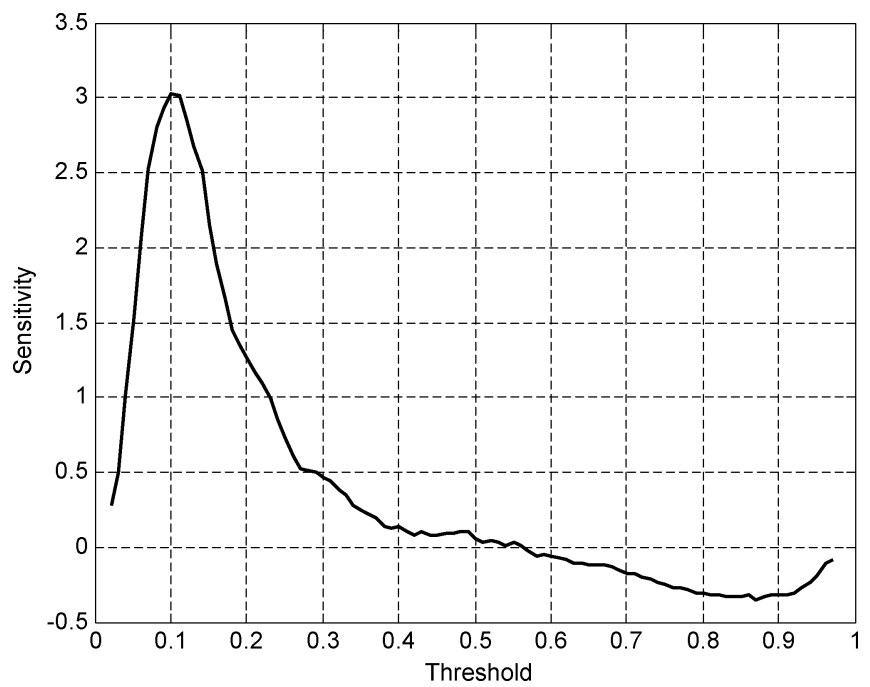

Fig. 4. Sensitivity of CCR to different threshold values.

TABLE I

PDFCC DECISION TABLE

\begin{tabular}{|c|c|c|}
\hline$C_{\text {animal }}$ & $C_{\text {tree }}$ & $y$ \\
\hline 0 & 0 & Others (O) \\
\hline 0 & 1 & Tree (T) \\
\hline 1 & 0 & Animal $(\mathrm{A})$ \\
\hline 1 & 1 & $\left\{\begin{array}{l}T,\left|P_{\text {tree }}-\zeta_{\text {tree }}\right| \geq\left|P_{\text {animal }}-\zeta_{\text {animal }}\right| \\
A,\left|P_{\text {animal }}-\zeta_{\text {animal }}\right|>\left|P_{\text {tree }}-\zeta_{\text {tree }}\right|\end{array}\right.$ \\
\hline
\end{tabular}

branches are consistent with each other, this decision fusion module simply combines the classification decisions from two branches as the final output. However, conflicting results do happen occasionally. For example, branch A claims that the input fault is caused by animal, while branch $\mathrm{T}$ claims it as tree-caused fault as well. When conflict occurs, this module makes a final classification decision by comparing the distances from the probability $P_{i}$ to the threshold $\zeta_{i}$ for both branches. The farther $P_{i}$ is from $\zeta_{i}$, the more likely that fault is caused by cause $i$. Since both ranges of the probability value $P_{i}$ for two branches are 1, we can directly compare the difference of $P_{i}$ and $\zeta_{i}$ without normalization. For example, if $\left|P_{i}-\zeta_{i}\right|>\left|P_{j}-\zeta_{j}\right|$, $i, j \in\{$ animal,tree $\}, i \neq j$, then the fault cause is categorized in class $i$.

\section{F. System Outputs}

The output of PDFCC indicates the cause type of the observed outage: $Y=\{y \mid y \in\{\operatorname{animal}(A), \operatorname{tree}(T)$, others $(O)\}\}$. The decisions are summarized in Table I.

\section{RESUlts AND Discussions}

The PDFCC uses outage data of seven selected Duke Energy's service regions from 1994 to 2002. The data for each region are divided into training data and test data: $3 / 4$ of the outage data for each region are randomly selected as training data, and the remaining $1 / 4$ of the data are used as test data. As an example, Clemson has 8376 valid outage records; 6282 of them are training data, and the remaining 2094 records are test data. 
TABLE II

CONFUSION MATRIX

\begin{tabular}{|c|c|c|}
\hline & $\begin{array}{c}\text { Predicted Positive } \\
\text { Class }\end{array}$ & $\begin{array}{c}\text { Predicted Negative } \\
\text { Class }\end{array}$ \\
\hline Actual Positive Class & True Positive (TP) & False Negative (FN) \\
\hline Actual Negative Class & False Positive (FP) & True Negative (TN) \\
\hline
\end{tabular}

\section{A. Performance Measures}

A straightforward way to measure the overall accuracy of a classifier is to test how many of the inputs are correctly classified into the corresponding categories, denoted by CCR. However, it is usually insufficient by simply observing CCR, especially when the data are imbalanced. For example, if a data set is composed of two classes, class $\mathrm{M}$ constitutes $95 \%$ of the data, while only $5 \%$ of the data are from class $\mathrm{N}$; then a classifier that predicts every case as the majority class $\mathrm{M}$ can still achieve a very high overall accuracy (95\%). As mentioned in Section IV, the power distribution outage data used are imbalanced in terms of both tree-caused faults and animal-caused faults. Therefore, true positive rate $A c c^{+}$, true negative rate $\mathrm{Acc}^{-}$, and geometric mean g-mean are further used to evaluate the performance of the techniques on the imbalanced data. These metrics are developed from the confusion matrix, as shown in Table II (assuming the animal/tree-caused faults are positive class and nonanimal/nontree faults as negative class).

True positive rate $\left(A c c^{+}\right)=T P /(T P+F N)$ indicates how many of the animal/tree-caused faults are correctly classified; true negative rate $\left(A c c^{-}\right)=T N /(T N+F P)$ shows how many of the nonanimal/nontree-caused faults are correctly classified. Kubat et al. propose the geometric mean of accuracies $(g-$ mean $)=\sqrt{\mathrm{Acc}^{+} \times A c c^{-}}$in [15] for imbalanced data sets. The basic idea behind g-mean is to maximize the accuracy on both classes; it is high when both $\mathrm{Acc}^{+}$and $\mathrm{Acc}^{-}$are large and the difference between $\mathrm{Acc}^{+}$and $\mathrm{Acc}^{-}$is small. Geometric mean punishes big disparities between accuracies of classifying animal/tree-caused faults and nonanimal/nontree-caused faults.

\section{B. Results}

As discussed in Section IV-E, the decision fusion module is to handle conflicting cases between two branches; in practice, only few conflicts occur (less than $0.1 \%$ of all the data encountered). We compare the performance for two individual branches instead of the overall PDFCC performance in order to provide detailed interpretation of the results, which are shown in Tables III and IV.

Both Table III and Table IV show that LR and ANN have similar performance in terms of the overall accuracy (CCR) for both animal-caused fault and tree-caused fault in all of seven selected service regions; ANN has slightly higher accuracy, though. From Table III, the largest difference between the performance of ANN and LR on animal-caused faults identification is 0.0131 for training data and only 0.0076 for test data. From Table IV, the largest difference between the performance of these two methods on tree-caused faults is 0.015 for training data and only 0.0089 for test data. The difference between these two techniques is so tiny that they are statistically insignificant. Besides, the difference of CCR between training data and test
TABLE III

CCR FOR ANIMAL FAULT CLASSIFIER WITH LR AND ANN

\begin{tabular}{|c|c|c|c|c|}
\hline & \multicolumn{2}{|c|}{ LR } & \multicolumn{2}{c|}{ ANN } \\
\hline region & Training data & Test data & Training data & Test data \\
\hline CHPL & 0.9028 & 0.8978 & 0.9158 & 0.9032 \\
\hline CLEM & 0.8603 & 0.8621 & 0.8697 & 0.8687 \\
\hline DURH & 0.9242 & 0.9227 & 0.9330 & 0.9288 \\
\hline GREE & 0.8829 & 0.8848 & 0.8944 & 0.8924 \\
\hline HICK & 0.8846 & 0.8787 & 0.8927 & 0.8828 \\
\hline LANC & 0.9348 & 0.9366 & 0.9385 & 0.9363 \\
\hline WINS & 0.9048 & 0.9014 & 0.9099 & 0.9023 \\
\hline
\end{tabular}

TABLE IV

CCR FOR TREE FAULT CLASSIFIER WITH LR AND ANN

\begin{tabular}{|c|c|c|c|c|}
\hline & \multicolumn{2}{|c|}{ LR } & \multicolumn{2}{c|}{ ANN } \\
\hline region & Training data & Test data & Training data & Test data \\
\hline CHPL & 0.8171 & 0.8098 & 0.8346 & 0.8172 \\
\hline CLEM & 0.7791 & 0.7770 & 0.7950 & 0.7859 \\
\hline DURH & 0.8486 & 0.8491 & 0.8630 & 0.8464 \\
\hline GREE & 0.7877 & 0.7860 & 0.8004 & 0.7884 \\
\hline HICK & 0.8367 & 0.8397 & 0.8525 & 0.8474 \\
\hline LANC & 0.7950 & 0.7864 & 0.8106 & 0.7938 \\
\hline WINS & 0.8346 & 0.8383 & 0.8455 & 0.8380 \\
\hline
\end{tabular}

data is also very small, which indicates that neither of the methods overfits the training data. The largest difference that LR gets on animal faults is just 0.0059 and on tree faults is 0.127 , while the largest difference that ANN gets on animal faults is only 0.0086 and on tree faults is 0.0174 .

We further use the animal fault classifier to illustrate true positive rate, true negative rate, and geometric mean, as shown in Figs. 5-7, respectively.

The true positive rate indicates the performance of two techniques on classifying the tree/animal faults. As shown in Fig. 5, ANN has better performance on this task; it outperforms the LR in all of the regions for training data and in five out of seven regions on test data. The largest difference between the two techniques is as large as $14.4 \%$ on training data and $11.8 \%$ on test data. Fig. 6 shows the true negative rate achieved by LR and ANN. Different from the case shown in Fig. 5 where ANN generally has higher true positive rate on both training data and test data, neither of these two methods shows obvious predominance; the largest difference between them is only $1.6 \%$ on training data and $2.1 \%$ on test data. Both advantages are gained by LR in the region of CLEM.

The imbalanced data typically cause the classifier to be biased toward the majority class (nontree/nonanimal fault), so as to have a low error rate for the majority class and get an unacceptable error rate for the minority class (tree/animal fault). Thus, we want to maximize the g-mean, which indicates the performance of PDFCC on classifying both fault causes. Fig. 7 indicates that ANN outperforms LR in most of the regions, the advantage is as large as 0.114 on training data and 0.091 on test data, and both occur in the region of DURH.

With the comparisons, ANN and LR have close overall classification accuracies. However, ANN achieves higher accuracy on classifying animal-caused faults than LR does, and it has a similar performance on classifying nonanimal-caused faults to LR's. Considering the animal fault is the minority class in our data and the nonanimal fault is the majority, LR is relatively 


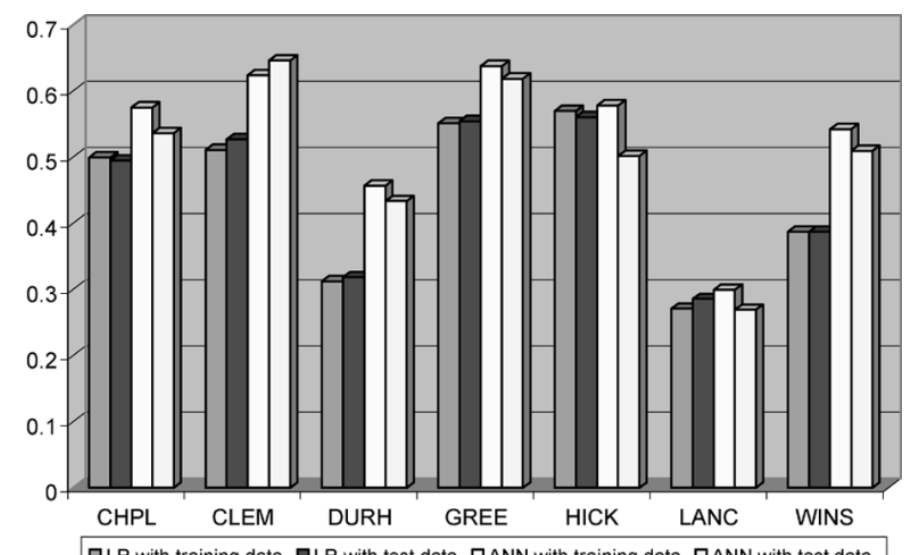

Fig. 5. True positive rate comparison.

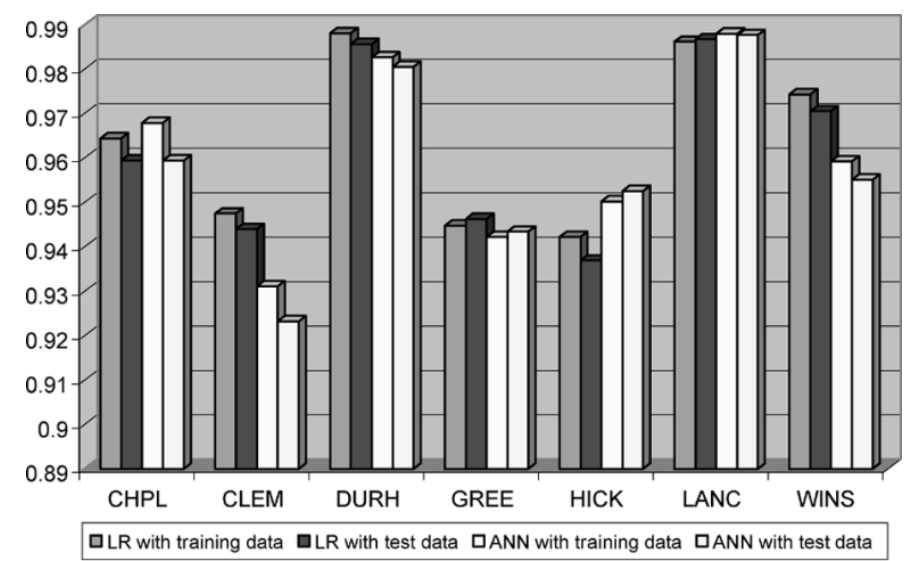

Fig. 6. True negative rate comparison.

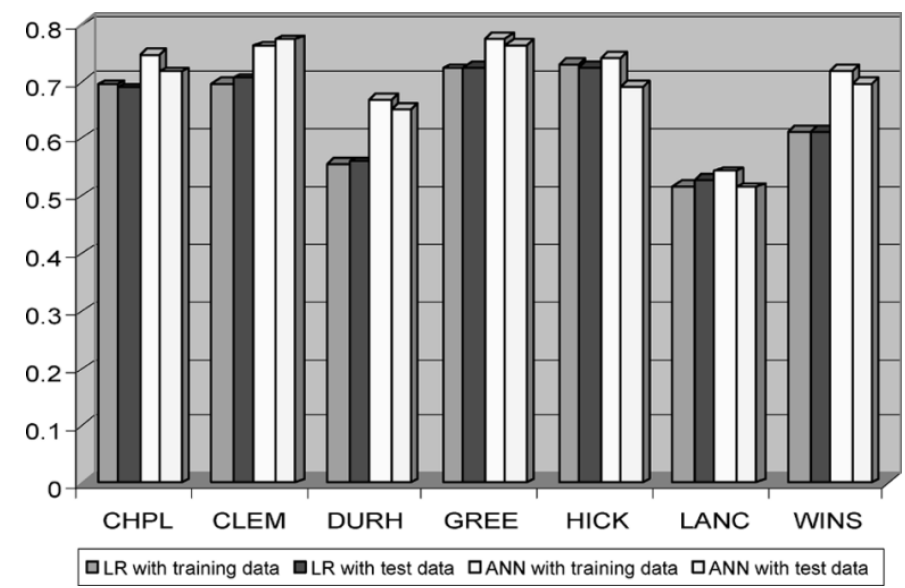

Fig. 7. g-mean comparison.

biased toward the majority data due to the imbalanced data constitution and results in a relatively worse accuracy for the minority class, which increases the disparities between accuracies of classifying two classes and then affects the g-mean.

\section{Discussions}

The Duke Energy outage data are used in this paper; in fact, the actual distribution outage data in most utilities are very noisy. The potential data quality problems (caused by the noise) include inconsistent data entries, inaccurate data entries, incomplete data entries, and insufficient input factors to distinguish two different types of faults. In our previous results reported in [14], we have filtered out all the noises, such as conflicting data, mis-entry, rare events (e.g., hurricane), etc., in order to focus on the technology development. As a result, we could achieve estimation accuracy of about $98 \%$ on animal-caused fault in the Durham region. In order to better reflect the real-world scenarios, we only filtered out the rare event data in this paper, while we left all other data (including conflicting data and inaccurate data) in the database. We achieved $93 \%$ estimation accuracy on animal-caused fault in the Durham region, which is comparable to an experienced power distribution engineer's best estimate on the fault cause identification.

The probability $P_{i}$ that we are expecting from the classification module of PDFCC can be actually expressed as $P\left(y=i \mid x_{1} \cap x_{2} \cap x_{3} \cap x_{4} \cap x_{5} \cap x_{6}\right)$, where $y$ denotes the power distribution fault cause $i \in\{$ animal,tree $\}$, while $x_{k}, k=1,2, \ldots, 6$ are the influential factors: CI, WE, NP, $\mathrm{PD}, \mathrm{SE}$, and TD. A straightforward way is to calculate this conditional probability directly from the historical data and then apply the calculated result on the test data. This approach will work, provided that enough data are available to calculate a statistically representative conditional probability $P_{i}$ given different event combinations $\left(x_{1} \cap x_{2} \cap x_{3} \cap x_{4} \cap x_{5} \cap x_{6}\right)$. In our outage data, $x_{1}(\mathrm{CI})$ has at least 30 circuit ID numbers included, this number differs from one region to another, $x_{2}$ (WE) includes 11 weather conditions, $x_{3}(\mathrm{PD})$ consists of 10 categories, $x_{4}$ (SE), $x_{5}$ (TD) and $x_{6}(\mathrm{NP})$ all have four possible events, so we need at least 211200 data samples to cover every single possible combination of different events, not to mention statistically representative probabilities calculation. However, the region with most outage records from these seven selected regions only includes less than 40000 outage data. So we will get a lot of zero probabilities and nonrepresentative conditional probabilities because of data shortage when using this approach. Alternatives such as approximate reasoning [16] have been investigated; in this paper, we calculate the likelihood measure $L$, which is the statistically representative conditional probability with respect to individual influential factor $x_{k}, k=1,2, \ldots, 6$ and then apply classification methods to implement the mapping from $L$ to desired $P_{i}$. This method successfully overcomes the difficulties due to data shortage and achieves satisfying results.

As mentioned in the introduction section, a regular LR is a parametric model that seldom is used in power system fault diagnosis, while ANN is a nonparametric method that has been extensively used in power systems. LR as a conventional statistical method has formalized models to exhibit the nonlinear relationship between the independent and dependent variables, while ANN can increase its flexibility by including hidden layers, which is often regarded as a substantial advantage of neural networks [17]. As seen from the results of PDFCC, ANN can achieve higher balanced accuracy than LR does. Both methods can be easily implemented by existing software; however, ANN requires relatively long training time and cross-validation requires even longer computational time, while LR is a much quicker method. 


\section{CONCLUSION}

Power distribution faults significantly affect the reliability and availability of the services provided by utilities; fast and proper outage restorations are essential to improve the quality of services and customer satisfaction. In this paper, we have developed a PDFCC to demonstrate the application of classification methods, LR and ANN, on the Duke Energy outage data for fault cause identification. Two major distribution fault types, tree and animal contact, are used as prototypes and four metrics, correct classification rate, true positive rate, true negative rate, and geometric mean, are employed to measure the performance with two techniques. This paper investigates some practical issues often faced in power distribution fault cause identification such as insufficient data and imbalanced data constitution as well as threshold setting. The advantages and disadvantages of the two techniques under these practical issues are also discussed.

\section{REFERENCES}

[1] A. Pahwa, "Role of distribution automation in restoration of distribution systems after emergencies," in Proc. IEEE Power Engineering Society Summer Meeting, vol. 1, 2001 , pp. 737-738.

[2] C.-F. Chien, S.-L. Chen, and Y.-S. Lin, "Using Bayesian network for fault location on distribution feeder," IEEE Trans. Power Del., vol. 17, no. 3, pp. 785-793, Jul. 2002.

[3] D. Thukaram, H. P. Khincha, and H. P. Vijaynarasimha, "Artificial neural network and support vector machine approach for locating faults in radial distribution systems," IEEE Trans. Power Del., vol. 20, no. 2, pp. 710-721, Apr. 2005.

[4] S.-J. Lee, M.-S. Choi, S.-H. Kang, B.-G. Jin, D.-S. Lee, B.-S. Ahn, N.-S Yoon, H.-Y. Kim, and S.-B. Wee, "An intelligent and efficient fault location and diagnosis scheme for radial distribution systems," IEEE Trans. Power Del., vol. 19, no. 2, pp. 524-532, Apr. 2004.

[5] O. Dag and C. Ucak, "Fault classification for power distribution systems via a combined wavelet-neural approach," in Proc. Int. Conf. Power System Technology, vol. 2, 2004 , pp. 1309-1314.

[6] K. L. Butler and J. A. Momoh, "A neural network based approach for fault diagnosis in distribution networks," in Proc. IEEE Power Engineering Society Winter Meeting, vol. 2, 2000, pp. 1275-1278.

[7] J.-T. Peng, C. F. Chien, and T. L. B. Tseng, "Rough set theory for data mining for fault diagnosis on distribution feeder," Proc. Inst. Elect. Eng., Gener., Transm., Distrib., vol. 151, no. 6, pp. 689-697, Nov. 2004.

[8] D. Niebur and A. J. Germond, "Power flow classification for static security assessment," in Proc. 1st Int. Forum Applications Neural Networks Power Systems, 1991, pp. 83-88.

[9] Y. Hayashi, S. Iwamoto, S. Furuya, and C.-C. Liu, "Efficient determination of optimal radial power system structure using Hopfield neural network with constrained noise," IEEE Trans. Power Del., vol. 11, no. 3, pp. 1529-1535, Jul. 1996.
[10] L. Xu, M.-Y. Chow, and L. S. Taylor, "Analysis of tree-caused faults in power distribution systems," in Proc 35th North American Power Symp., Oct. 20-21, 2003.

[11] M.-Y. Chow and L. S. Taylor, "Analysis and prevention of animal-caused faults in power distribution systems," IEEE Trans. Power Del., vol. 10, no. 2, pp. 995-1001, Apr. 1995.

[12] J. Han and M. Kamber, Data Mining: Concepts and Techniques. San Mateo, CA: Morgan Kaufmann, 2001.

[13] P. D. Allison, Logistic Regression Using the SAS System. Cary, NC: SAS Institute, 2000

[14] M.-Y. Chow, S. O. Yee, and L. S. Tayler, "Recognizing animal-caused faults in power distribution systems using artificial neural networks," IEEE Trans. Power Del., vol. 8, no. 3, pp. 1268-1274, Jul. 1993.

[15] M. Kubat, R. Holte, and S. Matwin, "Machine learning for the detection of oil spills in radar images," Mach. Learn., vol. 30, pp. 195-215, 1998.

[16] K. Tomsovic and B. Baer, "Methods of approximate reasoning for power system equipment condition and reliability analysis," in Proc. Intelligent Systems Applications Power Systems, 1996, pp. 310-315.

[17] M. Schumacher, R. Robner, and W. Vach, "Neural networks and logistic regression,” Comput. Stat. Data Anal., vol. 21, pp. 661-701, 1996.

[18] S. Santoso and J. D. Lamoree, "Power quality data analysis: From raw data to knowledge using knowledge discovery approach," in Proc. IEEE Power Engineering Society Summer Meeting, vol. 1, 2000, pp. 172-177.

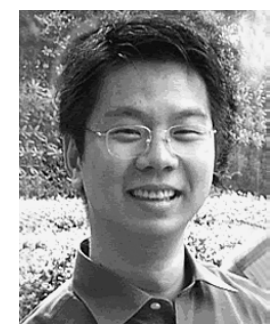

Le Xu (S'01) received the B.Eng. degree in automation from Tsinghua University, Beijing, China, in 2001 and the M.S. degree in electrical engineering from North Carolina State University (NCSU), Raleigh, in 2003. He is currently working toward the Ph.D. degree from the Advanced Diagnosis Automation and Control Laboratory, NCSU.

His research interests include intelligent health monitoring of power distribution systems.

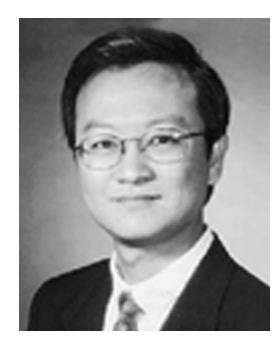

Mo-Yuen Chow (S'81-M'82-SM'93) received the B.S. degree in electrical and computer engineering from the University of Wisconsin, Madison, in 1982 and the M.Eng. and Ph.D. degrees from Cornell University, Ithaca, NY, in 1983 and 1987, respectively.

Upon completion of the Ph.D. degree, he joined the Department of Electrical and Computer Engineering, North Carolina State University (NCSU), Raleigh, and has held the rank of Professor since 1999. His core technology is diagnosis and control, artificial neural networks, and fuzzy logic with applications to areas including motors, process control, power systems, and communication systems. He established the Advanced Diagnosis Automation and Control Laboratory at NCSU. 